\title{
甲醇氧化PtSnCo/C 阳极催化剂
}

\author{
李庆武魏子栋”陈四国齐学强柳晓丁炜马 宇 \\ (重庆大学化学化工学院, 输配电装备及系统安全与新技术国家重点实验室, 重庆 400044)
}

\begin{abstract}
摘要: 通过乙二醇液相分步还原法制备了金属质量分数为 $20 \%$ 的 PtSn/C二元及 $\mathrm{PtSnCo} / \mathrm{C}$ 三元催化剂. 采用 $X$ 射线衍射 $(X R D)$ 光谱法、能量散射谱(EDS)对催化剂进行了表征; 通过阳极线性伏安扫描法 (LSV)、连续循环 伏安法 $(\mathrm{CV})$ 、预吸附单层 $\mathrm{CO}$ 溶出法研究了其电化学性质. 结果表明, $\mathrm{PtSnCo} / \mathrm{C}$ 三元催化剂较商业化 JM-PtRu/C 催化剂具有更好的氧化甲醇催化活性. 循环伏安扫描 100 圈后发现, PtSn/C二元催化剂的甲醇氧 化峰电流快速衰减到其初始氧化峰电流的 11\%左右, 而 PtSnCo/C 三元催化剂仅衰减到其初始值的 $50 \%$ 左右, 这表明 $\mathrm{PtSnCo} / \mathrm{C}$ 三元催化剂具有更好的化学稳定性. 在 $\mathrm{PtSnCo} / \mathrm{C}$ 催化剂上, 甲醇氧化起始电位比直接吸附 $\mathrm{CO}$ 后的 $\mathrm{CO}$ 阳极溶出电位负, 意味着甲醇在 $\mathrm{PtSnCo} / \mathrm{C}$ 催化剂上氧化的中间产物不是 $\mathrm{CO}$, 而是比 $\mathrm{CO}$ 更为活 泼且易于氧化的中间物种.
\end{abstract}

关键词: 直接甲醇燃料电池; PtSnCo/C; PtSn/C; 稳定性; 甲醇氧化 中图分类号: 0643.36

\section{PtSnCo/C Anode Catalyst for Methanol Oxidation}

\author{
LI Qing-Wu WEI Zi-Dong* CHEN Si-Guo QI Xue-Qiang LIU Xiao \\ DING Wei MA Yu
}

(State Key Laboratory of Power Transmission Equipment \& System Security and New Technology, School of Chemistry and Chemical Engineering, Chongqing University, Chongqing 400044, P. R. China)

\begin{abstract}
A binary metallic catalyst $(\mathrm{PtSn} / \mathrm{C})$ and a ternary metallic catalyst $(\mathrm{PtSnCo} / \mathrm{C})$ with a metal mass fraction of $20 \%$ were prepared by borohydride reduction and subsequent hydrothermal treatment in a glycol liquid phase. The structure and composition of the as-prepared electrocatalysts were characterized by X-ray diffraction (XRD) and energy-dispersive spectrometry (EDS). Their activity and stability for the catalysis of methanol oxidation were evaluated by anodic linear sweep voltammetry (LSV), cyclic voltammetry (CV), and the anodic stripping of a pre-adsorbed $\mathrm{CO}$ monolayer. We found that the $\mathrm{PtSnCo} / \mathrm{C}$ catalyst gave the best catalytic activity for the methanol oxidation of all the catalysts including the commercial JM-PtRu/C catalyst. After 100 cycles, the peak current of methanol oxidation for the PtSn/C catalyst rapidly decreased to $11 \%$ of its initial peak current but $\mathrm{PtSnCo} / \mathrm{C}$ decreased to only $50 \%$. This result suggests that the $\mathrm{PtSnCo} / \mathrm{C}$ catalyst has better chemical stability for the catalysis of methanol oxidation compared to the PtSn/C catalyst. The more negative onset potential of methanol oxidation for the $\mathrm{PtSnCo} / \mathrm{C}$ catalyst relative to pre-adsorbed $\mathrm{CO}$ oxidation implies that the intermediates of methanol oxidation on the $\mathrm{PtSnCo} / \mathrm{C}$ catalyst may be ones, which can be more easily oxidized than $\mathrm{CO}$, instead of $\mathrm{CO}$.
\end{abstract}

Key Words: Dierct methanol fuel cell; $\mathrm{PtSnCo} / \mathrm{C} ; \mathrm{PtSn} / \mathrm{C}$; Stability; Methanol oxidation

\footnotetext{
Received: May 4, 2011; Revised: August 25, 2011; Published on Web: September 5, 2011.

"Corresponding author. Email: zdwei@cqu.edu.cn; Tel: +86-23-65105160.

The project was supported by the National Natural Science Foundation of China (20906107, 20936008), Innovative Talent Training Project, Chongqing University, China (101061136), and Fundamental Research Funds for the Central University, China (CDJXS10221141, 11132229). 国家自然科学基金(20906107, 20936008), 重庆大学 985 创新人才培养建设计划(101061136)和中央高校基础研究基金(CDJXS10221141, 11132229)资助项目
}

(C) Editorial office of Acta Physico-Chimica Sinica 


\section{1 引言}

直接甲醇燃料电池(DMFC)因其具有质量轻、 体积小、结构简单、比能量密度高、低温操作等优 点, 在移动电源、电动汽车等领域具有广泛前景. ${ }^{1,2}$ 但困扰 DMFC 商业化发展的三个主要问题是: ${ }^{3}$ (1) 甲醇在阳极上氧化反应速率缓慢; (2) 甲醇自阳极透 过质子交换膜向阴极渗透使电池阴极性能下降; (3) 甲醇氧化产物 $\mathrm{CO}_{2}$ 的液封效应问题. 发展性能优异 的催化剂是克服甲醇氧化反应速率缓慢的最有效 手段. 在酸性溶液中, 甲醇氧化最有效的催化剂是 $\mathrm{Pt}$ 或 $\mathrm{Pt}$ 基合金催化剂. 然而, 甲醇氧化反应产生的 $\mathrm{CO}$ 中间体强吸附在 Pt 表面形成 Pt- $\mathrm{CO}_{\text {ads }}$ 而使其失 活. 一般认为, 4,5 铂电极只有在相对参比氢电极 (RHE) 在 $0.5 \mathrm{~V}$ 以上才能形成含氧物种使 $\mathrm{CO}_{\mathrm{ads}}$ 进一 步氧化为 $\mathrm{CO}_{2}$, 从而使其恢复活性. 因此, 提高 $\mathrm{Pt}$ 对 甲醇氧化反应活性的关键是在低电位下高效地氧 化去除 $\mathrm{CO}$ 以释放出 $\mathrm{Pt}$ 的活性位. ${ }^{6}$

目前的解决办法是在 Pt中加入一种或几种能 在低电位下生成含氧物种的金属形成二元或多元 的 $\mathrm{Pt}$ 基合金催化剂. ${ }^{7.8} \mathrm{PtRu} / \mathrm{C}$ 是公认的商业化效果 最好的 DMFC 阳极电催化剂. $\mathrm{Ru}$ 原子促进 $\mathrm{CO}$ 氧化 的双功能机理已被普遍认可, ${ }^{9-12}$ 即在比 $\mathrm{Pt}$ 电位低 $0.2-0.3 \mathrm{~V}$ 的电位下, $\mathrm{Ru}$ 表面能形成含氧物种, 从而 实现在较低的电位下氧化去除 CO 中间体. Goodenough 等 ${ }^{13}$ 提出 $\mathrm{PtRu}$ 中 $\mathrm{Pt}$ 电子结构的改变是其抗 $\mathrm{CO}$ 中毒能力改善的另一种解释. PtSn 是仅次于 $\mathrm{PtRu}$ 而倍受关注的另一个二元催化剂. 有研究报道 在 $\mathrm{Pt}$ 上欠电位沉积 $\mathrm{Sn}$ 原子且 $\mathrm{Sn}$ 的覆盖率在 $20 \%$ 左 右时, 发现其对 $\mathrm{Pt}$ 在较低电位下发生的甲醇氧化反 应表现出了明显的催化增强作用, 认为 $\mathrm{Sn}$ 的作用也 同样可以用双功能机理和电子效应来解释. ${ }^{14,15}$ 然 而, 锡对铂的甲醇氧化催化活性是否具有增强作用 还持有争议. 如 Frelink 等 ${ }^{16}$ 发现 PtSn 合金催化剂并 没有表现出比 Pt 更优异甚至更差的甲醇氧化催化 活性, 有报道认为这是由于锡的存在不利于 $\mathrm{Pt}$ 上的 甲醇吸附以及脱氢过程. ${ }^{17}$ 此外, 酸性燃料电池操作 条件下 PtSn 合金催化剂中 Sn 易于溶解脱出, 因此, $\mathrm{PtSn}$ 二元合金催化剂催化甲醇氧化稳定性还有待 进一步提高. PtRuSn, ${ }^{18} \mathrm{PtRuCo},{ }^{19} \mathrm{PtSnNi}{ }^{20}$ 等三元合 金催化剂相继被报道, 均发现三元合金催化剂具有 更好的甲醇氧化催化活性、抗 CO 中毒能力以及电 化学稳定性. Travitsky 等 ${ }^{21}$ 通过热酸处理形成 $\mathrm{Pt}_{74} \mathrm{CoSn}_{26}$ 近似于核-壳结构的三元催化剂, 用于直接
甲醇燃料电池正极氧还原催化且有耐透醇能力, 即 只对氧还原有催化作用, 对甲醇氧化无催化作用. 然而 Beyhan 等 ${ }^{22}$ 使用原位红外光谱观察到 PtSnCo 催化乙醇氧化时不仅较纯 $\mathrm{Pt}$ 上吸附了更少的类 $\mathrm{CO}$ 中间体以及乙酸等吸附物, 而且在乙醇氧化过程中 产生更多的 $\mathrm{CO}_{2}$, 表明与纯 $\mathrm{Pt}$ 相比, $\mathrm{PtSnCo}$ 对乙醇 等小分子有机物具有更高的催化活性. 可见, 三元 催化剂 PtSnCo 是否具有催化小分子醇氧化的能力 因合成方法、原子间比、结构状态不同而不同.

本文通过乙二醇液相分步还原法制备了金属 质量分数为 $20 \%$ 的 $\mathrm{PtSnCo} / \mathrm{C}$ 三元催化剂. 采用 XRD、EDS 对催化剂进行了物理化学表征, 通过阳 极线性伏安扫描法、预吸附单层 CO 溶出法、连续循 环伏安法研究了其电化学性质. 特别探讨了 Co 的加 入对 $\mathrm{PtSn} / \mathrm{C}$ 二元催化剂的电化学性质的影响, 其结 果对改善 $\mathrm{PtSn} / \mathrm{C}$ 二元催化剂的性能有启示意义.

\section{2 实验部分}

\section{1 试剂与仪器}

如无特别说明所用试剂均为分析纯: 硝酸钴 $\left(\mathrm{Co}_{2}\right.$ $\left.\left(\mathrm{NO}_{3}\right)_{3} \cdot 9 \mathrm{H}_{2} \mathrm{O}\right)$ 、氯化亚锡 $\left(\mathrm{SnCl}_{2} \cdot 2 \mathrm{H}_{2} \mathrm{O}\right)$ 、浓硫酸, 浓盐 酸均为重庆无机化学试剂厂产品; 嗍氢化钠 $\left(\mathrm{NaBH}_{4}\right.$, 天津市化学试剂研究所); 柠檬酸三钠(TCD), 甲醇、 无水乙醇、甲酸、氢氧化钠(重庆川东化学试剂厂); 氯铂酸 $\left(\mathrm{H}_{2} \mathrm{PtCl}_{6} \cdot 6 \mathrm{H}_{2} \mathrm{O}\right.$, 上海精细化工材料研究所); 氮气(重庆泰和气体实业有限责任公司, 99.999\%); 商业化 vulcan X72 碳粉 (美国 Cabot Corp.); 20\% $\mathrm{Pt}_{50} \mathrm{Ru}_{50} / \mathrm{C}, 40 \% \mathrm{Pt} / \mathrm{C}$ 催化剂 (美国 Johnson-matthey Corp.); $0.5 \%$ Nafion (美国 Dupont Corp); 所有的电 解质溶液均用超纯水 $(18.2 \mathrm{M} \Omega \cdot \mathrm{cm})$ 来配制.

碳纸 TGP-H-090 (日本 Torry Corp 公司); 自压 反应釜 RD-50 (中国石油化工股份有限公司石油化 工科学研究院); 电化学工作站 AutoLab (荷兰, Metrohm Singapore Pte Ltd.); XRD-6000 型 X射线衍射 仪(日本岛津公司); FEI Nova 400 型扫描电子显微 镜(Peabody, MA, Netherland).

\section{2 催化剂的制备}

Vulcan XC-72 碳粉的预处理按照文献 ${ }^{23}$ 进行, 催化剂制备具体过程如下: 取 $80 \mathrm{mg}$ 预处理后的 Vulcan XC-72 碳粉, $5.2 \mathrm{mg} \mathrm{SnCl}_{2} \cdot 2 \mathrm{H}_{2} \mathrm{O}, 20 \mathrm{mg}$ $\mathrm{Co}\left(\mathrm{NO}_{3}\right)_{2} \cdot 9 \mathrm{H}_{2} \mathrm{O}$ 和一定量的 TCD (TCD 和金属摩尔 比为 2:1)加入到 $20 \mathrm{~mL}$ 乙二醇中, 超声搅拌 $0.5 \mathrm{~h}$ 使 金属盐充分溶解于乙二醇中, 调节 $\mathrm{pH} \sim 11$, 然后在强 
烈搅拌下将 $5 \mathrm{~mL}$ 浓度为 $8 \mathrm{~g} \cdot \mathrm{L}^{-1}\left(\mathrm{NaBH}_{4} /\right.$ 乙二醇)溶 液滴加到上述体系中, 摚拌反应 $1 \mathrm{~h}$ 后使 $\mathrm{Sn}^{2+}$ 和 $\mathrm{Co}^{2+}$ 还原于碳粉上, 再向上述溶液中加入 $0.7 \mathrm{~mL}$ 浓度为 $40 \mathrm{~g} \cdot \mathrm{L}^{-1}$ 的 $\mathrm{H}_{2} \mathrm{PtCl}_{6}$ 水溶液(保持 $\mathrm{Pt}: \mathrm{Sn}$ :Co 的原子比为 $3: 1: 3)$, 调节 $\mathrm{pH} 11$, 分散后转移入自压反应釜中, $160{ }^{\circ} \mathrm{C}$ 下高温反应 $6 \mathrm{~h}$, 取出自然降温后, 抽滤, 用 1 : 1 无水乙醇/水混合溶液洗涤数次, 除掉氯离子和多 余的 TCD, $60{ }^{\circ} \mathrm{C}$ 真空干燥 $24 \mathrm{~h}$ 后待用. 所制备的催 化剂标记为 $\mathrm{PtSnCo} / \mathrm{C}$. 使用相同的方法及步骤制备 $\mathrm{PtSn} / \mathrm{C}$ 催化剂, 催化剂中 $\mathrm{Pt}: \mathrm{Sn}$ 的理论原子比为 $3: 1$.

\section{3 催化剂的物理化学性质表征}

催化剂晶相结构和晶粒大小的分析是在 XRD$6000 \mathrm{X}$ 射线衍射仪上进行的, 衍射源为 $\mathrm{Cu} K_{a}$ 靶 $(\lambda=$ $0.1542 \mathrm{~nm}$ ), 测试角度为 $10^{\circ}-80^{\circ}$, 扫描速率为 $2\left(^{\circ}\right)$. $\mathrm{min}^{-1}$. 催化剂上 Pt 纳米颗粒的平均粒径可以通过 Scherrer 公式计算.

$$
d=0.9 \lambda /(B \cdot \cos \theta)
$$

上式中 $\lambda$ 为入射光波长 $(0.1542 \mathrm{~nm}), B$ 是以弧度表示 的衍射峰半峰宽(FWHM), $\theta$ 为衍射峰出现位置的角 度. $d$ 为引起该衍射晶面的法线方向上的晶面尺寸 (nm). 对于晶体平均粒径大小的计算, 一般来讲, 如 是较大颗粒的晶体则应该选择较高晶面指数的半 峰宽; 相反, 如果较小细微晶体, 则应该选择较低晶 面指数的半峰宽. 同时, 尽量选择受干扰小的晶面 特征峰的半峰宽. 本文选择 $\operatorname{Pt}(111)$ 和 $\operatorname{Pt}(200)$ 两个晶 面特征峰的平均值来估算 $\mathrm{Pt}$ 晶粒平均粒径大小.

EDS 表征是在 FEI Nova 400 扫描电镜下进行, 选取三个区域取其平均值来计算催化剂中 Pt、Sn、 Co 的原子比.

\section{4 电催化性能评价}

采用线性扫描伏安法(LSV)、循环伏安法(CV) 和预吸附单层 $\mathrm{CO}$ 溶出法研究催化剂的电化学性 能, 并与英国 Johnson-Matthey 公司的商业 $\mathrm{Pt} / \mathrm{C}$ 催化 剂(记为 $\mathrm{JM}-\mathrm{Pt} / \mathrm{C}$ ) 进行比较. 电化学测试采用三电极 体系在 AutoLab 电化学工作站上进行, 其中辅助电 极为铂丝, 参比电极为 $\mathrm{Ag} / \mathrm{AgCl}$ (饱和 $\mathrm{KCl}$ 溶液) 电 极 (本文电位值无特别说明均是相对于 $\mathrm{Ag} / \mathrm{AgCl}$ 电 极电位). 电解质分别为 $0.5 \mathrm{~mol} \cdot \mathrm{L}^{-1} \mathrm{H}_{2} \mathrm{SO}_{4}$ 溶液以及 $0.5 \mathrm{~mol} \cdot \mathrm{L}^{-1} \mathrm{H}_{2} \mathrm{SO}_{4}+0.5 \mathrm{~mol} \cdot \mathrm{L}^{-1} \mathrm{CH}_{3} \mathrm{OH}$. 电化学测试 中持续向电解质中通入氮气以驱除溶解氧, 所有测 试均在室温下进行.

预吸附单层 $\mathrm{CO}$ 溶出实验. 先向电解质中通高 纯氮 $0.5 \mathrm{~h}$ 除氧, 以 $50 \mathrm{mV} \cdot \mathrm{s}^{-1}$ 在 $-0.15-1.05 \mathrm{~V}$ 范围
内扫描 10 圈使电极表面达到稳定, 再向电解质中通 入 $\mathrm{CO}$ (实验室甲酸和浓硫酸制备 $\mathrm{CO}$ ) $0.5 \mathrm{~h}$, 使电极 表面上 $\mathrm{CO}$ 的吸附达到饱和, 停止通 $\mathrm{CO}$, 再用高纯 氮除去溶液中的 $\mathrm{CO}$, 然后进行循环伏安扫描, 扫描 速率为 $50 \mathrm{mV} \cdot \mathrm{s}^{-1}$.

工作电极制备: 称取 $2 \mathrm{mg}$ 催化剂, 加入 $400 \mu \mathrm{L}$ 无水乙醇和适量 $0.5 \%$ Nafion, 超声波振荡 30-60 $\min$, 混合至成均匀墨水状液体, 取 $100 \mu \mathrm{L}$ 悬浮液涂 于按照文献 ${ }^{24}$ 处理的 TGP-H-090 碳纸上 $(1 \mathrm{~cm} \times 1.5$ $\mathrm{cm})$, 涂平, $80^{\circ} \mathrm{C}$ 烘干, 即得到工作电极. 相应催化 剂制备的电极, 记为相应的电极, 如 $\mathrm{PtSn} / \mathrm{C}$ 催化剂 制备的电极记为 $\mathrm{PtSn} / \mathrm{C}$ 电极.

\section{3 结果与讨论}

\section{1 催化剂的XRD及EDS 表征}

图 1 是各催化剂的 XRD 图. 从图 1 可以看出在 $2 \theta$ 为 $40.4^{\circ} 、 44.5^{\circ} 、 68.2^{\circ}$ 处分别对应 $\mathrm{Pt}$ 的(111)、(200)、 (220)晶面衍射峰. 与标准谱图(JCPDS-652868)对比 发现, $\mathrm{PtSn} / \mathrm{C}$ 和 $\mathrm{PtSnCo} / \mathrm{C}$ 催化剂均为 $\mathrm{Pt}$ 的面心立方 晶系. 在衍射图谱中没有出现 $\mathrm{Sn} 、 \mathrm{SnO}_{2} 、 \mathrm{Co} 、 \mathrm{CoO}_{2}$ 、 $\mathrm{Co}_{3} \mathrm{O}_{4}$ 等特征衍射峰, 推测 $\mathrm{Sn}$ 或 $\mathrm{Co}$ 在催化剂中是以 无定形态或固溶体形式存在. 根据 XRD 估算的 $\mathrm{JM}-\mathrm{Pt} / \mathrm{C}, \mathrm{PtSn} / \mathrm{C}, \mathrm{PtSnCo} / \mathrm{C}$ 的平均粒径分别为 3.1、 $3.2 、 2.3 \mathrm{~nm}$.

对上述催化剂的 EDS 分析发现, $\mathrm{PtSn} / \mathrm{C}$ 催化剂 中 Pt:Sn 原子比为 3.53:1, 基本接近制备时的配料 比. 但 $\mathrm{PtSnCo} / \mathrm{C}$ 催化剂 Pt:Sn:Co 原子比为 4.06:1.26: 1 (见图 2), Pt:Sn 原子比基本接近于制备时的配料 比, Co 的含量远低于理论值. 我们推测这是因为 $\mathrm{Co}^{2+}$ 是三种金属离子里是最难被还原的, 还原速率

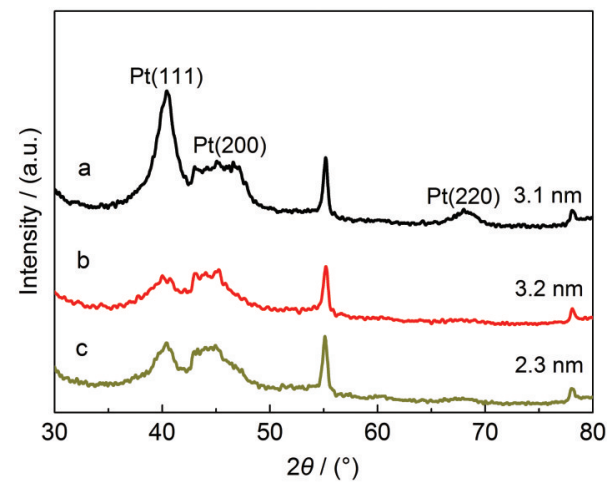

图 $1 \mathrm{JM}-\mathrm{Pt} / \mathrm{C}(\mathrm{a}) 、 \mathrm{PtSnCo} / \mathrm{C}(\mathrm{b})$ 和 PtSn/C (c) 粉末催化剂 的 XRD 谱

Fig.1 XRD patterns of JM-Pt/C (a), PtSnCo/C (b), and $\mathrm{PtSn} / \mathrm{C}$ (c) catalyst powder 


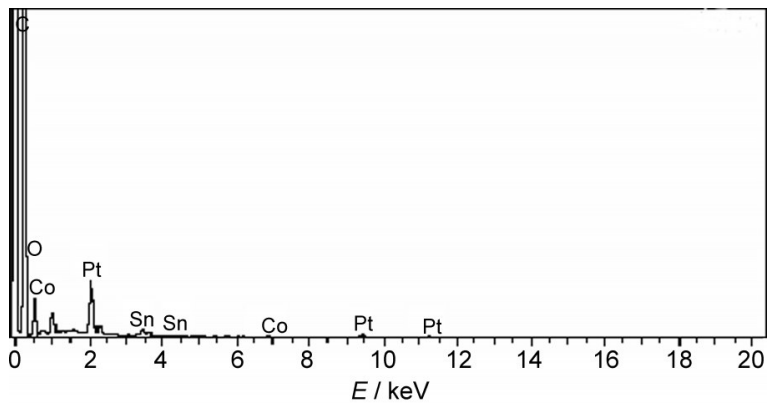

图 $2 \mathrm{PtSnCo} / \mathrm{C}$ 催化剂的 $\mathrm{EDS}$ 分析

Fig.2 EDS spectra of PtSnCo/C Catalyst

的不一致导致了 $\mathrm{Co}$ 偏离配料值. 此外, 柠檬酸三钠 的络合作用进一步导致 $\mathrm{Co}^{2+}$ 难以还原.

\section{2 催化剂的电化学测试}

图 3 是各电极在 $0.5 \mathrm{~mol} \cdot \mathrm{L}^{-1} \mathrm{H}_{2} \mathrm{SO}_{4}+0.5 \mathrm{~mol}$ $\mathrm{L}^{-1} \mathrm{CH}_{3} \mathrm{OH}$ 溶液阳极线性扫描伏安曲线. 从图 3 看出 $\mathrm{PtSn} / \mathrm{C}$ 电极的甲醇氧化起始电位与商业化的 JM-Pt/ $\mathrm{C}$ 电极基本一致, 并没有明显的提升甲醇氧化反应 的活性, 表明 $\mathrm{Sn}$ 的加入并没有增强 $\mathrm{Pt}$ 的甲醇氧化催 化活性, 这与 Frelink 等 ${ }^{16}$ 的观察现象是一致的. 然 而, 当形成 $\mathrm{PtSnCo} / \mathrm{C}$ 三元催化剂后, 其甲醇氧化起 始电位比 $\mathrm{PtSn} / \mathrm{C}$ 电极明显负移了约 $150 \mathrm{mV}$, 与商业 化 JM-PtRu/C 电极的甲醇氧化起始电位一致, 但其 峰电流密度是所有电极中最高的, 说明 $\mathrm{Co}$ 的加入有 效提升了 $\mathrm{PtSnCo} / \mathrm{C}$ 电极的甲醇氧化催化活性.

通常认为, 甲醇氧化过程的主要中间体是 $\mathrm{CO}$. 图 4 是各电极上预吸附 $\mathrm{CO}$ 后的阳极溶出实验. 从图 4 中可以看出 $\mathrm{JM}-\mathrm{Pt} / \mathrm{C}$ 上 $\mathrm{CO}$ 氧化起始电位在 $0.62 \mathrm{~V}$ 左右, 与文献报道值 $0.7 \mathrm{~V}(v s \mathrm{RHE})^{25}$ 接近. $\mathrm{PtSn} / \mathrm{C}$ 的 $\mathrm{CO}$ 氧化起始电位与 $\mathrm{JM}-\mathrm{Pt} / \mathrm{C}$ 相比负移了约 $100 \mathrm{mV}$

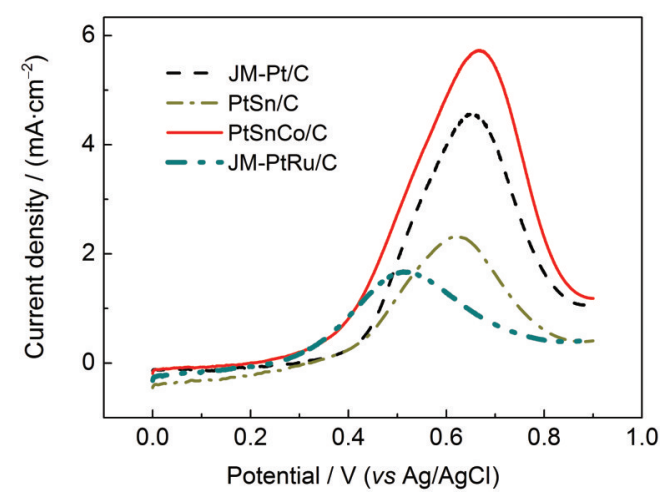

图 3 各电极在 $0.5 \mathrm{~mol} \cdot \mathrm{L}^{-1} \mathrm{H}_{2} \mathrm{SO}_{4}+0.5 \mathrm{~mol} \cdot \mathrm{L}^{-1} \mathrm{CH}_{3} \mathrm{OH}$ 电 解液中的线性伏安曲线

Fig.3 Anodic linear sweep voltammograms of the methanol electrooxidation reaction on different electrodes in $0.5 \mathrm{~mol} \cdot \mathrm{L}^{-1} \mathrm{H}_{2} \mathrm{SO}_{4}+0.5 \mathrm{~mol} \cdot \mathrm{L}^{-1} \mathrm{CH}_{3} \mathrm{OH}$ electrolyte scan rate: $5 \mathrm{mV} \cdot \mathrm{s}^{-1}$

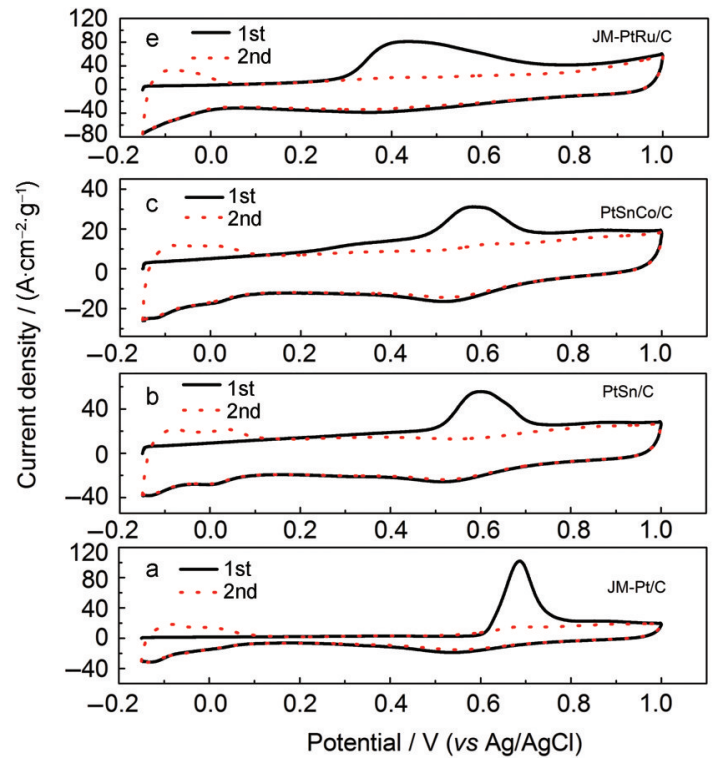

图 4 在 $0.5 \mathrm{~mol} \cdot \mathrm{L}^{-1} \mathrm{H}_{2} \mathrm{SO}_{4}$ 溶液中不同电极上预吸附单层 CO 的溶出伏安曲线

Fig.4 Anodic stripping voltammograms of pre-adsorbed $\mathrm{CO}$ monolayer on differenct electrodes in $0.5 \mathrm{~mol} \cdot \mathrm{L}^{-1}$ $\mathrm{H}_{2} \mathrm{SO}_{4}$ solution

scan rate: $50 \mathrm{mV} \cdot \mathrm{s}^{-1} .1$ st cycle (black solid line) for the electrooxidation of $\mathrm{CO}, 2 \mathrm{nd}$ cycle (red dot line) after removal of $\mathrm{CO}$

(0.5 V 左右), 表明其比铂更容易在低电位下催化氧 化 CO. 这是因为 $\mathrm{Sn}$ 能在比 $\mathrm{Pt}$ 更低的电位下形成 $\mathrm{Sn}-\mathrm{OH}_{\mathrm{ads}}$ 物种, ${ }^{17}$ 从而降低 $\mathrm{CO}$ 的电位. 但与商业化 $\mathrm{JM}-\mathrm{PtRu} / \mathrm{C}(0.3 \mathrm{~V}$ 左右 $)$ 相比, $\mathrm{PtSn} / \mathrm{C}$ 的氧化 $\mathrm{CO}$ 的电 位明显偏高. 而 $\mathrm{PtSnCo} / \mathrm{C}$ 的 $\mathrm{CO}$ 氧化起始电位与 $\mathrm{PtSn} / \mathrm{C}$ 相比略负, 但不及商业化 JM-PtRu/C 的 CO氧 化起始电位负. 说明 $\mathrm{Co}$ 的加入并不能进一步降低 $\mathrm{PtSn} / \mathrm{C}$ 催化剂的 $\mathrm{CO}$ 氧化电位. 图 3 和图 4 的结果显 示, 在 $\mathrm{JM}-\mathrm{PtRu} / \mathrm{C}$ 电极上, 甲醇和 $\mathrm{CO}$ 的起始氧化电 位基本一致, 大约 $0.3 \mathrm{~V}$. 而在 $\mathrm{Pt} / \mathrm{C} 、 \mathrm{PtSn} / \mathrm{C} 、 \mathrm{PtCo} / \mathrm{C}$ 、 $\mathrm{PtSnCo} / \mathrm{C}$ 电极上, 甲醇氧化起始电位均比直接吸 附 $\mathrm{CO}$ 后的 $\mathrm{CO}$ 阳极溶出电位负, 很可能是甲醇在 这些电极上氧化的中间产物是比 $\mathrm{CO}$ 更为活泼且 易于氧化的中间物种, 如 $\mathrm{CHO}(\mathrm{COH}) 、 \mathrm{CH}_{3} \mathrm{O} 、 \mathrm{HCO}$ 、 $\mathrm{CH}_{2} \mathrm{O},{ }^{26-28}$ 或者甲醇氧化产生的吸附态 $\mathrm{CO}$ 并不完全 等同于从 $\mathrm{CO}$ 气体吸附到催化剂表面的 $\mathrm{CO}$. 类似的 现象在孙世刚等 ${ }^{29}$ 的研究中也有观察. Co 加入后, $\mathrm{PtSnCo} / \mathrm{C}$ 电极相对于 $\mathrm{Pt} / \mathrm{C} 、 \mathrm{PtSn} / \mathrm{C} 、 \mathrm{PtCo} / \mathrm{C}$ 电极甲醇 氧化电流显著增加, 是晶粒细化, 电极表面活性位 增加所致(见图 $1 \mathrm{XRD}$ 表征的晶粒尺度).

图 5 是 $\mathrm{PtSn} / \mathrm{C}$ 和 $\mathrm{PtSnCo} / \mathrm{C}$ 电极分别在 $0.5 \mathrm{~mol}$ $\mathrm{L}^{-1} \mathrm{H}_{2} \mathrm{SO}_{4}+0.5 \mathrm{~mol} \cdot \mathrm{L}^{-1} \mathrm{CH}_{3} \mathrm{OH}$ 溶液中的连续循环伏 安曲线, 记录了 100 圈的实验数据(如图 $5(\mathrm{a}, \mathrm{b})$ ). 通 

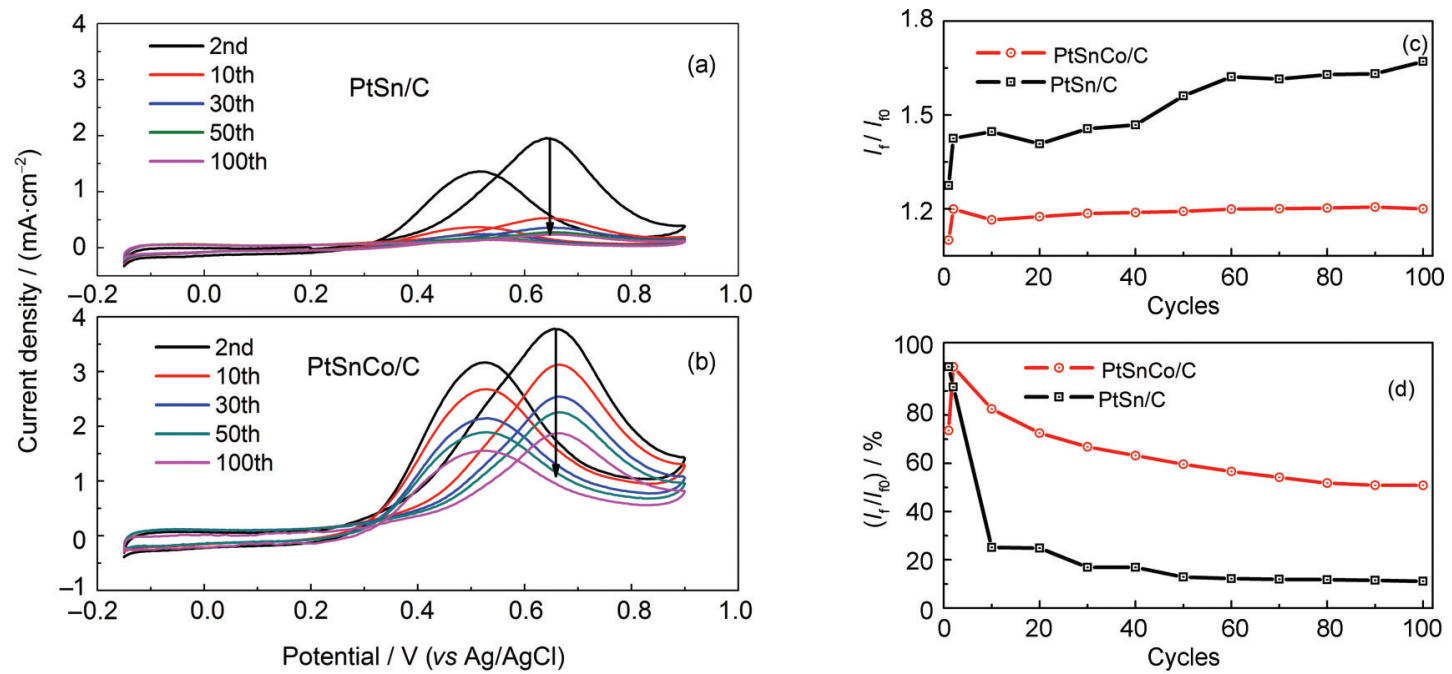

图 $5 \mathrm{PtSn} / \mathrm{C}$ 和 $\mathrm{PtSnCo} / \mathrm{C}$ 电极在 $0.5 \mathrm{~mol} \cdot \mathrm{L}^{-1} \mathrm{H}_{2} \mathrm{SO}_{4}+0.5 \mathrm{~mol} \cdot \mathrm{L}^{-1} \mathrm{CH}_{3} \mathrm{OH}$ 溶液中的连续循环伏安曲线图 $(\mathrm{a}, \mathrm{b})$ 以及峰电流 $I_{\mathrm{f}} / I_{\mathrm{b}}(\mathrm{c})$ 和 $I_{\mathrm{f}} / I_{\mathrm{f} 0}(\mathrm{~d})$ 随扫描圈数的变化

Fig.5 Cyclic voltammograms (a, b) of electrodes PtSn/C and PtSnCo/C recorded in $0.5 \mathrm{~mol} \cdot \mathrm{L}^{-1} \mathrm{H}_{2} \mathrm{SO}_{4}+0.5 \mathrm{~mol} \cdot \mathrm{L}^{-1} \mathrm{CH}_{3} \mathrm{OH}$ electrolyte with $\mathrm{N}_{2}$ bubbled at scan rate: $10 \mathrm{mV} \cdot \mathrm{s}^{-1}$, ratio of $I_{\mathrm{f}} / I_{\mathrm{b}}(\mathrm{c})$ and $I_{\mathrm{f}} / I_{\mathrm{f} 0}(\mathrm{~d})$ with cycle numbers on two electrodes $I_{\mathrm{f}}$ : the forward peak current, $I_{\mathrm{b}}$ : the backward peak current, $I_{\mathrm{t}}$ : the initial current of the forward current

过对连续循环伏安扫描过程中催化剂的甲醇氧化 正向峰电流与反向峰电流的比值 $I_{\mathrm{f}} I_{\mathrm{b}}$ 分析发现(图 $5 \mathrm{c}), \mathrm{PtSnCo} / \mathrm{C}$ 和 $\mathrm{PtSn} / \mathrm{C}$ 的 $I_{\mathrm{f}} / I_{\mathrm{b}}$ 的初始值基本相同, 但 $\mathrm{PtSnCo} / \mathrm{C}$ 三元催化电极的 $I_{\mathrm{f}} / I_{\mathrm{b}}$ 的值随扫描圈数的 增加而基本不变, 进一步说明其具有更好的电化学 稳定性. ${ }^{30-32}$ 虽然 $\mathrm{PtSn} / \mathrm{C}$ 电极的 $I_{\mathrm{f}} / I_{\mathrm{b}}$ 值随扫描圈数增 加而逐渐增大, 似乎意味着表面毒性中间体氧化电 流相对甲醇正向氧化电流越来越少. 但是, 图 5(d)显 示, 经过 100 圈连续循环伏安扫描后, $\mathrm{PtSn} / \mathrm{C}$ 电极的 甲醇氧化峰电流 $I_{\mathrm{f}}$ 衰减到初始氧化峰电流 $I_{\mathrm{f}}$ 的 $11 \%$ 左右, 而 $\mathrm{PtSnCo} / \mathrm{C}$ 电极仅衰减到初始值的 $50 \%$ 左 右, 说明 $\mathrm{PtSn} / \mathrm{C}$ 电极的活性位 (面积) 显著下降, $\mathrm{PtSnCo} / \mathrm{C}$ 电极却具有更好的化学稳定性.

\section{4 结 论}

探讨了 $\mathrm{Co}$ 的加入对 $\mathrm{PtSn} / \mathrm{C}$ 二元催化电极的电 化学性质的影响. 结果表明, (1) PtSnCo/C 三元催化 剂具有与商业化 $\mathrm{PtRu} / \mathrm{C}$ 催化剂相近的甲醇氧化催 化活性, 与 $\mathrm{PtSn} / \mathrm{C}$ 二元催化剂相比具有更高的催化 甲醇氧化活性和稳定性. $\mathrm{Co}$ 加入后, $\mathrm{PtSnCo} / \mathrm{C}$ 催化 剂相对于 $\mathrm{Pt} / \mathrm{C} 、 \mathrm{PtSn} / \mathrm{C} 、 \mathrm{PtCo} / \mathrm{C}$ 催化剂, 甲醇氧化电 流显著增加, 是晶粒细化, 电极表面活性位增加的 结果. (2) 在 $\mathrm{PtSnCo} / \mathrm{C}$ 催化剂上, 甲醇氧化起始电位 比直接吸附 $\mathrm{CO}$ 后的 $\mathrm{CO}$ 阳极溶出电位负, 意味着甲 醇在 $\mathrm{PtSnCo} / \mathrm{C}$ 催化剂上氧化的中间产物是比 $\mathrm{CO}$ 更
为活泼且易于氧化的中间物种, 或者不完全等同于 从 $\mathrm{CO}$ 气体吸附到催化剂表面的类 $\mathrm{CO}$ 物种. 考虑到 锡、钴比钓价廉, 三元催化剂 $\mathrm{PtSnCo} / \mathrm{C}$ 作为甲醇氧 化阳极催化剂前景更好.

\section{References}

(1) Prater, K. B. J. Power Sources 1996, 61, 105.

(2) Doo, H. J.; Chang, H. L.; Chang, S. K.; Dong, R. S. J. Power Sources 1998, 71, 169.

(3) Xu, Q. J.; Zhou, X. J.; Li, Q. X.; Li, J. G. Acta Phys. -Chim. Sin. 2010, 26, 2135. [徐群杰, 周小金, 李巧霞, 李金光. 物理化学 学报, 2010, 26, 2135.]

(4) Beden, B.; Lamy, C.; Bewick, A.; Kunimatsu, K. J. Electroanal. Chem. 1981, 121, 343.

(5) Hamnett, A. Catal. Today 1997, 38, 445.

(6) Li, L. L.; Wei, Z. D.; Yan, C.; Luo, Y. H.; Yin, G. Z.; Sun, C. X. Acta Phys. -Chim. Sin. 2007, 23 (5), 723. [李兰兰, 魏子栋, 严 灿, 罗义辉, 尹光志, 孙才新. 物理化学学报, 2007, 23 (5), 723.]

(7) An, X. S.; Chen, D. J.; Zhou, Z. Y.; Wang, Q.; Fan, Y. J.; Sun, S. G. Acta Phys. -Chim. Sin. 2010, 26, 1207. [安笗莎, 陈德俊, 周志有, 汪 强, 㚞友军, 孙世刚. 物理化学学报. 2010, 26, 1207.]

(8) Wei, Z. D.; Guo, H. T.; Tang, Z. Y. J. Power Sources 1996, 58 , 239.

(9) Chrzanowski, W.; Kim, H.; Wieckowski, A. Catal. Lett. 1998, 50,69 .

(10) Wei, Z. D.; Li, L. L.; Luo, Y. H.; Yan, C.; Sun, C. X.; Yin, G. Z.; Shen, P. K. J. Phys. Chem. B 2006, 110, 26055. 
(11) Chrzanowski, W.; Wieckowski, A. Langmuir 1997, 13, 5974.

(12) Zhou, W. J.; Zhou, Z. H.; Li, W. Z.; Sun, G. Q.; Xin, Q. Chemistry 2003, 66 (4), 228. [周卫江, 周振华, 李文震, 孙公权, 辛 勤. 化学通报. 2003, 66 (4), 228.]

(13) Goodenough, J. B.; Manoharan, R.; Shukla, A. K.; Ramesh, K. V. Chem. Mater. 1989, 1, 391.

(14) Wei, Z. D.; Miki, A.; Ohmori, T.; Osawa, M. Acta Phys. -Chim. Sin. 2002, 18 (12), 1120. [魏子栋, 三木敦史, 大森唯义, 大泽 雅致. 物理化学学报, 2002, 18 (12), 1120.]

(15) She, C. X.; Li, X. Q.; Ren, B.; Lin, H. S.; Tian, Z. Q. Chinese Journal of Light Scattering 2002, 3, 223. [余春兴, 李筏琴, 任 斌, 林华水, 田中群. 光散射学报, 1999, 3, 223]

(16) Frelink, T.; Visschefz, W.; Van Veen, J. A. R. Electrochim. Acta 1994, 39, 1871

(17) Antolini, E.; Gonzalez, E. R. E. Catal. Today 2011, 160, 28.

(18) Neto, A. O.; Dias, R. R.; Tusi, M. M.; Lindardi, M.; Spinacé, E. V. J. Power Sources 2007, 166, 87.

(19) Strasser, P. J. Comb. Chem. 2008, 10, 216.

(20) Spinacé, E. V.; Lindardi, M.; Neto, A. O. Electrochem. Commun. 2005, 7, 365.

(21) Travitsky, N.; Burstein, L.; Rosenberg, Y.; Peled, E. J. Power Sources 2009, 194, 161.

(22) Beyhan, S.; Kadirgan, F.; Léger, J. M. In-situ Infrared Spectroscopy Study of Ethanol Oxidation on Pt and PtSn-Based
Trimetallic Anode Electrocatalysts for Direct Ethanol Fuel Cell. In Electrode Processes Relevant to Fuel Cell Technology. 217th ECS Meeting, Vancouver, Canada, April 25-30, 2010; Birss, V.; Kulesza, P.; Mustain, W.; Ota, K.; Wilkinson, D.; The Electrochemical Society 2010, B7, 603.

(23) Wei, Z. D.; Chen, S. G.; Liu, Y.; Sun, C. X.; Shao, Z. G.; Shen, P. K. J. Phys. Chem. C 2007, 111, 15456.

(24) Liao, M. J.; Wei, Z. D.; Chen, S. G.; Li, L.; Ji, M. B.; Wang, Y. Q. Int. J. Hydrog. Energy 2010, 35, 8071.

(25) Crabb, E. M.; Marshall, R.; Thompsett, D. J. Electrochem. Soc. 2000, 147,4440 .

(26) Xia, X. H.; Iwasita, T.; Ge, F.; Vielstich, W. Electrochim. Acta 1996, $41,711$.

(27) Iwasita, T.; Braz. J. Chem. Soc. 2002, 13, 401.

(28) Wang, J.; Masel, R. I. Surf. Sci. 1991, 235, 199.

(29) Zhou, Z. Y.; Tian, N.; Zeng, D. M.; Sun, S. G. The Proceeding of 12th National Conference of Electrochemistry, Shanghai, China, 2003, A040. [周志有, 田 娜, 曾冬梅, 孙世刚. 第 12 次全国电化学会议论文集, 上海, 2003, A040.]

(30) Chen, W.; Kim, J.; Sue, S.; Chen, S. Langmuir 2007, 23, 11303.

(31) Chen, J.; Wang, M.; Liu, B.; Fan, Z.; Cui, K.; Kuang, Y. J. Phys. Chem. B 2006, 110, 1775.

(32) Hsieh, C. T.; Lin, J. Y. J. Power Sources 2009, 188, 347. 